\title{
Desarrollo local: conceptualizaciones, principales características y dimensiones.
}

Local development: conceptualizations, main characteristics and dimensions.

\author{
MSc. Dagmar Santa Cruz Pérez ${ }^{1}$, Dra.C Victoria Ojalvo Mitrany. ${ }^{2}$ \\ \& Dr.C. Efraín Velasteguí López ${ }^{3}$
}

Recibido: 10-11-2018 / Revisado: 17-02-2019 /Aceptado: 25-02-2019/ Publicado: 04-04-2019

\begin{abstract}
.
DOI: https://doi.org/10.33262/cienciadigital.v9i2.353

The study addresses in the first place a characterization of the development of local development in Cuba and the different obstacles and advances that have materialized over time, as well as the challenges that still need to be achieved based on the felt and recognized needs of the communities and the opportunities that can be achieved, based on the recognition of the social actors involved and the political will of local governments, as well as the postures that have been brought to bear on different conceptions of local development by the authors who have developed it.

It is necessary to visualize all the characteristics and dimensions that local development can pay for the responsible knowledge of the actors in the different areas of action, in search of possible solutions to the problems of the community where the elements of integration and participation are key in the development of local development dynamics. The objective of this study is not only to describe the state of the art of local development and its development in Cuba, but also to prepare the way for the current conditions of local development demanded by the community and the autonomy delegated to local governments. in the Cuban context, all about the perception of the AGENDA 2030.
\end{abstract}

Keywords: Local Development, conceptions, dharacteristics, dimensions and dynamics.

\footnotetext{
${ }^{1}$ Universidad de la Habana, Rectorado, La Habana, Cuba, dstacruz@rect.uh.cu

${ }^{2}$ Universidad de la Habana, CEPES, La Habana, Cuba, victoria@cepes.uh.cu

${ }^{3}$ Ciencia Digital, Ecuador, Ambato, luisefrainvelastegui@cienciadigital.org
} 


\section{Resumen.}

El estudio aborda en primer orden una caracterización del desenvolvimiento del desarrollo local en Cuba y los diferentes obstáculos y avances que se han podido materializar a través del tiempo, así como los retos que todavía quedan por lograr a partir de las necesidades sentidas y reconocidas de las comunidades y las oportunidades que se puedan lograr, a partir del reconocimiento de los actores sociales implicados y la voluntad política de los gobiernos locales, además de las posturas aterrizadas acerca de diferentes concepciones de desarrollo local por los autores que la han desarrollado.

Se hace necesario visualizar todas las características y dimensiones que el desarrollo local puede tributar para el conocimiento responsable de los actores en los diferentes espacios de acción, en busca de posibles soluciones a los problemas de la comunidad donde los elementos de integración y participación son claves en el desenvolvimiento de la dinámica de desarrollo local. El objetivo de este estudio no solo radica en describir el estado del arte del desarrollo local y su desenvolvimiento en Cuba, sino el hecho de, preparar el camino para las condiciones actuales de desarrollo local que exige la comunidad y la autonomía delegada a los gobiernos locales en el contexto cubano, todo sobre la percepción de la AGENDA 2030.

Palabras claves: Desarrollo local, concepciones, características, dimensiones, dinámica.

\section{Introducción}

En el análisis crítico de experiencias de desarrollo local en Cuba a escala nacional, se evidencia que se han tenido notables avances en la senda del desarrollo sostenible, pero en el ámbito local lo alcanzado está por debajo de lo que la realidad exige; esto fundamenta la necesidad de seguir trabajando en tan noble propósito. La experiencia más actual, es la Estrategia de desarrollo local sostenible, en el municipio de Los Palacios, al suroeste de la Provincia de Pinar del Rio, liderada por Gorgoy Lugo, Director de Proyecto Consejo Administración Provincial Pinar del Río, Coordinador Provincial de la Plataforma Articulada para el Desarrollo Integral Territorial. Compendio de buenas prácticas para el desarrollo local en América Latina la cual se desarrolló en el periodo 2013/2014 y en la actualidad se encuentra en fase de implementación.

(www.oecd.org/cfe/leed/summerschool)(C OCDE 2016)

La estrategia busca contribuir a la toma de decisiones que definan las estrategias, políticas, programas y proyectos de desarrollo territorial, a partir de la implicación de los actores locales en el aprovechamiento de los recursos endógenos y de la articulación entre intereses nacionales, sectoriales y territoriales. En Cuba, existen otras iniciativas locales incorporadas a la senda del desarrollo sostenible.

Mencionemos la de Santa María del Rosario, comunidad periférica situada al Noroeste del municipio de El Cotorro y al sudoeste de La Habana. Este proyecto se inserta, a escala global, en la iniciativa internacional de Comunidades Saludables acordada por la Asamblea Mundial de la Salud, y en el ámbito local surge como una idea propia de la comunidad. La elaboración e implementación de un programa de esta naturaleza, según sus autores, con énfasis en la 
relación salud, medio ambiente y participación comunitaria, intentan potenciar el desarrollo y la participación ciudadana para hacer compatible la conservación y uso sostenible de los recursos naturales con la salud de la población. Otra experiencia es la del Proyecto de Apoyo a la Agenda 21 de la ciudad de Bayamo, (Proyectos de Granma en www.pdhl.onu.org.cu), insertado en el Programa de las Naciones Unidas para el desarrollo de Cuba, que pretende fortalecer las capacidades de autoridades locales y los actores claves del desarrollo para enfrentar la temática urbana ambiental, mediante la programación participativa integrada. En este caso, el énfasis se hace en el medio ambiente físico natural, pero el desarrollo sostenible tiene que tener un enfoque multidimensional, que integre las diferentes dimensiones del proceso de sostenibilidad. Otra de las iniciativas de carácter local en Cuba,es el estudio realizado en las localidades de Villa Clara para diagnosticar los diferentes niveles de desarrollo de estas, centrando la atención en la mortalidad infantil, una de las variables de la dimensión social y en el orden económico, en el índice de ocupación y en la productividad del trabajo.

En la ciudad de Santiago de Cuba se están dando los primeros pasos para elaborar un proyecto que permita un desarrollo sostenible. En el resto del territorio, el proceso de desarrollo local que se ejecuta en el ámbito municipal resulta insuficiente, por cuanto los aspectos relacionados con el desarrollo local,se tratan de forma parcial, sin lograr una integración de los procesos claves de la gestión económica, social y ambiental; se requiere, entonces, un perfeccionamiento de la orientación hacia la sostenibilidad, que permita su integración y mejoramiento en el ámbito del municipio.

La valoración del autor del estudio es que el desarrollo local en Cuba es una disciplina en desarrollo donde están aterrizando en la actualidad, muchas experiencias interesantes en diversas comunidades y sus estudios están siendo registrados y monitoreados por varias instituciones docentes como por ejemplo la Facultad latinoamericana de Ciencias Sociales (FLACSO) de la Universidad de La Habana (UH).

Aunque su concepto se hace difícil de comprender desde la interdisciplinaridad, y verlo fusionado con otros elementos tan importantes para su desarrollo y perfeccionamiento, como la comunicación y la perspectiva desde la ciencia, tecnología e innovación, esto no quiere decir que Cuba dentro de sus políticas públicas no siga visualizando una estrategia bien pensada al respecto, lo que falta es el sentido de articulación e integración para lograr procesos acabados de transformación social con una reciprocidad constante activa y sistemática con todos los actores sociales y la comunidad como principal protagonista del proceso.

Este aterrizaje entendido como una unión indisolublees la que va a tributar resultados sorprendentes desde la base en la comunidad hasta la cima de los resultados de esas políticas públicas. Es por eso que hay que seguir identificando todo tipo de desaciertos, errores, insuficiencias, desconocimiento, entre otros, para seguir mitigando el problema, trabajando a su vez a partir de la principal fortaleza que se tiene: la comunidad, que es la que define el proceso, lo optimiza, lo regula y lo perfecciona. 
De ahí es que este estudio cultiva sus cimientos articulados a partir de la comprensión de la educación, la comunicación, el desarrollo local y la perspectiva de ciencia, tecnología e innovación, como elementos claves en los procesos de transformación social en comunidades concebidas a partir de la articulación de actores.

Se suma a este análisis el valor y papel de la educación, la cual constituye hoy día un factor esencial del desarrollo social y económico ampliamente reconocido y un reto inaplazable para toda la sociedad, por las profundas y constantes transformaciones a que se encuentra sometida bajo la influencia de la revolución científica-técnica-productiva y las crecientes demandas de la población.

Esta situación impone no pocos cambios en todos los campos de acción relacionados con la formación constante y continúa de los recursos humanos, tal y como se refrendara en la Declaración de la Conferencia de Cartagena (CRES), en la que al analizar el contexto de la región se estableció que: "en un mundo donde el conocimiento, la ciencia y la tecnología juegan un papel de primer orden, el desarrollo y el fortalecimiento de la Educación

Superior constituyen un elemento insustituible para el avance social, la generación de riqueza, el fortalecimiento de las identidades culturales, la cohesión social, la lucha contra la pobreza y el hambre, la prevención del cambio climático y la crisis energética, así como para la promoción de una cultura de paz”(UNESCO, 2008, pág. 41).

\section{Desarrollo}

El desarrollo local y comunitario constituye un eslabón importante en el desarrollo sociocultural de la sociedad, la Organización de las Naciones Unidas (ONU) se ha pronunciado de manera enfática y profunda al respecto, al considerar que el sentimiento de identidad con la comunidad, con sus propósitos y objetivos, es una precondición para generar y sustentar el desarrollo de la comunidad.

Se trata de un proceso destinado a crear condiciones de progreso económico y social para toda la comunidad, con la participación real de sus actores en el mejoramiento de su nivel de vida.

En dependencia de su propia iniciativa, de la articulación de actores y del desarrollo de prácticas comunicativas con fines educativos se marcarán pautas significativas y definitorias. Al desarrollo local y comunitario lo integran, según la posición de dicho organismo internacional, los procesos por medio de los cuales los esfuerzos del pueblo mismo se unifican con los de las autoridades para mejorar las condiciones económicas, sociales y culturales de las comunidades, para integrarlas a la vida nacional, de forma que contribuyan también al desarrollo de la nación.

Este conjunto de procesos según la Unión Europea, (U. E. 2014) está constituido por les indicadores esenciales como la participación de los habitantes en los esfuerzos por mejorar su nivel de vida, en dependencia de sus ideas, iniciativas y propósitos, y la prestación tanto 
de servicios técnicos como de otra índole, de manera que se estimule la iniciativa, la autoayuda y la colaboración mutua, con vistas a hacerlos mucho más eficaces.

Según ambos indicadores, el desarrollo local y comunitario como proceso posee una gran importancia en el sentido organizacional y educativo: organizacional, porque requiere la reorientación de las instituciones existentes o la creación de nuevos tipos de instituciones, y educativo porque implica el cambio de actitudes y prácticas que obstaculizan las mejoras socioeconómicas. El desarrollo local ha sido tratado en la literatura especializada como proceso, como metodología y también como técnica, incluyendo sus procesos de gestión.

La Comisión Europea define el desarrollo local desde la comunidad como un enfoque que da la vuelta a la política tradicional de desarrollo descendente. (Unión Europea, 2014). El problema de las comunidades ha llamado la atención de los organismos internacionales. La Organización de las Naciones Unidas, la Organización de los Estados Americanos y la Organización Mundial de la Salud, se han pronunciado en relación con el desarrollo y la salud comunitarios y han elaborado planes para fomentarlos.

En 1948, la Conferencia Africana sobre Administración se pronunció sobre la relación condiciones de vida-educación del pueblo, y como consecuencia de ello, a partir de 1950 comenzaron a aparecer numerosas publicaciones sobre comunidad, estudios comunitarios, desarrollo de la comunidad, organización de la comunidad, etc. Son muchas las ocasiones en las que aparecen conceptos en las investigaciones que están sometidos a discusión por parte del cuerpo científico.

El "desarrollo local" es uno de ellos, sobre todo si tenemos en cuenta las modificaciones que sufre al relacionarse con la esfera global.

El concepto de "desarrollo local" surge de la combinación de dos términos controvertidos, que han sido objeto de discusión científica y han generado posturas dispares. Se han realizado distintas aportaciones para ofrecer una definición unánime y aceptada que todavía no se ha conseguido. El concepto de desarrollo ha sido utilizado en la mayoría de las ocasiones con una significación cualitativa. En el lenguaje común se emplea como sinónimo de crecimiento, y generalmente, aumento económico.

Por otro lado, la noción de "local" hace referencia a una parte del espacio, pequeña y englobada o referenciada en una estructura más amplia. Ambos son conceptos relacionados íntimamente y han tenido un protagonismo creciente en el cuerpo científico del siglo XXI.

El concepto ha sido abordado desde diversas perspectivas, áreas y autores por lo que se dificulta lograrel consenso sobre su definición. Se ha visto como "proceso de transformación y promoción social, económica o cultural" o como "la mejora de los procesos de comunicación para lograr una mayor inteligencia social” (Llobera, 2001. pág., 53). Se asume que existe una relación estrecha con otros procesos. 
Esta conexión genera problemas cuando desarrollo se entiende como una asimilación de los términos "progreso" y "evolución" e incluye, además, algunos sinónimos ("adelanto", "crecimiento", "ampliación" o "mejora") que complican su descripción. El desarrollo viene marcado por su contexto histórico (Arocena, 2002).

Fue A. Touraine el que extendió la noción de desarrollo para aplicarlo a la historia y lo utilizó como categoría de análisis válida, en ese caso, para los procesos de industrialización. "Desenvolverse" o "desarrollarse" significó recorrer un camino predeterminado gracias a un conjunto de leyes naturales, que contrastaba con la "reconstrucción" que las potencias avanzadas tenían que realizar después de la II Guerra Mundial, y que dejaba diseñado, de alguna forma la nueva manera de organizar el espacio en el mundo (Touraine, 1995).

El espacio local ha sido considerado, por muchos autores, el lugar donde comenzar a solucionar los problemas ypara conseguirlo de manera efectiva a escala global. Es la parcela en la que los esfuerzos de planificación y organización deben ser aplicados, es la zona desde la que se debe construir una base sólida de desarrollo, que permita extrapolarlo a un ámbito cada vez mayor. Esta premisa conduce a considerar que este espacio local está compuesto por ámbitos diversos.

Lo conforman redes sociales, actitudes políticas, territorio, dinámicas económicas y un largo etcétera, que puede generar un sistema bien organizado.

Lo "local”, referido al desarrollo, es una dimensión que supera la territorialidad geográfica, es relevante en la medida que afecta a un "espacio de convivencia" en el que se construyen las relaciones humanas (Valdizán, 2007, p.269). Esta dimensión se asume como proceso cuando se opta por una acción intencional de desarrollo para lograr una identidad. El desarrollo dentro de lo local, antes mencionado, se puede entender como la oportunidad para recibir nuevas influencias y trasladar experiencias. Según Dollfus (1997) el ámbito local es:

- Lo que se sitúa en determinadas coordenadas terrestres,

- lo que caracteriza a un lugar, y cada lugar se define por su posición en el planeta, su situación en relación con otros lugares con los que establece relaciones, su emplazamiento, que es su soporte físico.

- Además, lo local implica el espacio en el que se vive más permanentemente, donde se duerme, donde se trabaja, donde se frecuenta a los allegados, el espacio de lo cotidiano, de vecindad, formado por lugares utilizados y frecuentados a diario.

- Asimismo, es uno de los niveles de participación en la vida del ciudadano (p. 61)

Silva (2006) citada en Carbajal (2011) anota que tal vez uno de los rasgos sobresalientes sea la emergencia de la dimensión espacial como referente fundamental de los procesos económicos, políticos y sociales. 
El territorio, como soporte de la actividad humana, pasa a ser también un factor explicativo esencial de los procesos de desarrollo. La importancia del territorio, la localidad, del lugar, va a ser relevante en procesos del llamado desarrollo local. Arocena (2002) señala que referirse a lo local no está exento de ambigüedad, de impresiones, de dificultades de definición.

Para definir la noción local no hay otro camino que referirla a su noción correlativa de global. Cuando algo se define como local es porque pertenece a lo global. Esta primera constatación tiene que llevar a una aseveración categórica: nunca se puede analizar un proceso de desarrollo local sin referirlo a la sociedad global en la que está inscrito Arocena (2002). Este autor se refiere en sus estudios sobre procesos de desarrollo local a la importancia de su dimensión identitaria.

En este sentido se hace necesario acotar dos apreciaciones importantes: en la primera se argumenta que en la esfera local se genera la convivencia con el territorio, se hace referencia a los valores, tendencias y actitudes de la vida cotidiana, haciendo partícipe a la comunidad, al territorio y al espacio de las actividades, decisiones y posturas dialogadas en consenso con la suma de las necesidades conscientemente reconocidas, o no, con énfasis en la participación popular, mostrando el poder del lugar, entendido como elemento global donde se pueden expresar las relaciones de los distintos elementos que lo componen.

La segunda se refiere, según la apreciación del autor del estudio, a lo local, es el ámbito donde la gente vive, piensa, crea, coordina y expone lo vivido, donde se relaciona con el medio que le rodea y donde se crean los poderes sociales.

No es posible la existencia de procesos exitosos de desarrollo local sin un componente identitario fuerte, que estimule y vertebre el potencial de iniciativas de un grupo humano. Una definición de lo local según Arocena (2002) debe tomar distancia al mismo tiempo del aislacionismo autárquico y del reduccionismo globalizante.

Es necesario entonces adoptar una serie de precauciones, se trata de un concepto relativo, es una respuesta a un estado de la sociedad, supone una definición de actor social bien precisa, parte de una concepción integral e integradora del desarrollo, se sitúa al mismo tiempo en la afirmación de lo singular y de las regularidades estructurales. Arocena (2002) refiere que el desarrollo local no es una tecnología a aplicar en cualquier circunstancia, es, ante todo, una estrategia sociopolítica de cambio.

Algunos de los aspectos que plantea Silva (2006) citado en Carbajal (2011), se asemejan a los de muchos de los autores antes mencionados, por lo que se percibe una coincidencia al caracterizar los procesos de desarrollo local, que se expresan a continuación:

1. Son procesos de naturaleza endógena.

2. Buscan su estrategia en una solidaridad con el territorio. 
3. Responden a una voluntad de gestión partenarial o asociativa entre representantes públicos y privados.

4. Poseen liderazgo y animación del proceso.

5. La originalidad de las experiencias locales consiste en que permiten recoger y estimular todos los elementos endógenos y dinámicos desde una perspectiva integral.

6. Son difícilmente ajustables a modelos o estructuras de gestión muy rígidas o cerradas.

7. Poseen acciones que se pueden comprender y que se transforman en características específicas de estos procesos. Veamos la figura 1.

Figura1. Sinergia como proceso continuo hacia el desarrollo local

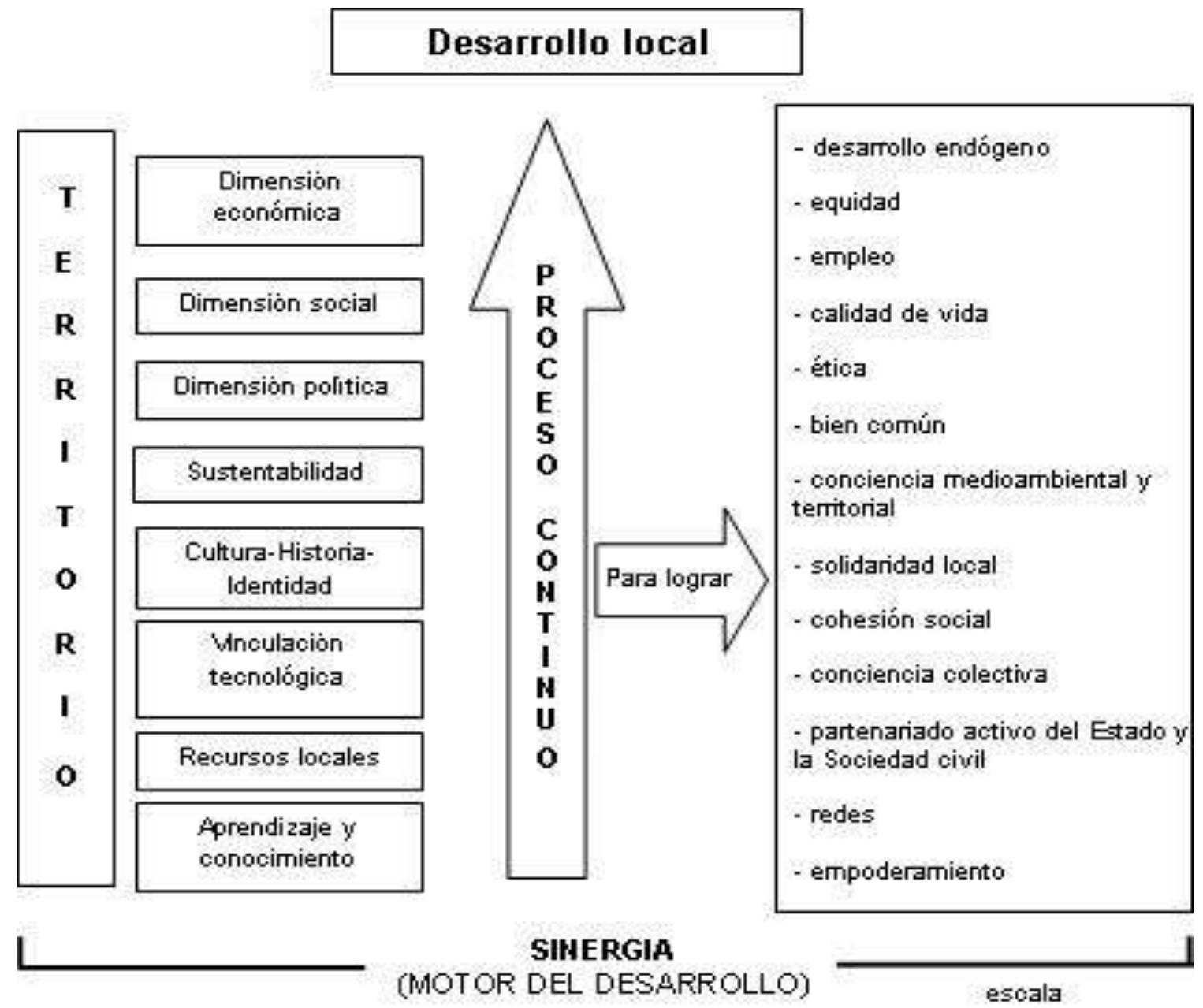

Fuente: http://www.ecured.cu/index. Php /Desarrollo—local

Cotorruelo (2003, p.2) se refiere y lo asocia a "un proceso de objetivos múltiples, que son: eficiencia en la asignación de recursos para la competencia territorial; equidad en la distribución de la renta y equilibrio del entorno medioambiental para la conservación del sistema productivo territorial" En el contexto del método de desarrollo local se considera la 
participación como un proceso en el que la comunidad se compromete con la transformación de su propia realidad y asume las tareas que le corresponden.

De esta manera el movimiento de su transformación deja de ser una suma de aventuras individuales para expresarse colectivamente bajo la forma de proyectos solidarios o propuestas comunes.

Se trata de un largo proceso de aprendizaje de nuevas relaciones sociales, de nuevos modos de comportamiento, de una nueva visión de las cosas. Corvalan y Ferreira (2003, p.16-17), citado en Carbajal (2011). Estos autores identifican ocho principios que orientan las acciones de desarrollo local:

1. El método es participativo, se construye en base a la cooperación entre pares y el diálogo entre los actores comprometidos.

2. Promueve las acciones a partir del autoconocimiento de la realidad local y de análisis crítico. Para esto es preciso hacer converger dos miradas, una mirada local y una mirada técnica donde todos aprenden. El técnico tiene una información amplia, maneja datos, sabe compararlos y organizarlos, confronta sus teorías con la realidad y las reajusta o las rectifica si es necesario.

3. La comunidad revela ámbitos desconocidos, informa sobre lo que los técnicos ignoran, aporta su visión concreta y vivida. Por otro lado, aprende a considerar nuevos horizontes. Las dos miradas se complementan, se reestructuran, se articulan para dar origen a un nuevo modo de ver las cosas.

4. El técnico escucha antes de orientar. No impone caminos, acompaña y enseña a caminar.

5. Identifica intereses comunes para acciones organizadas en el marco de un proyecto colectivo.

6. Fortalece a la comunidad, impulsando acciones organizadas y buscando relaciones con sus semejantes hasta la constitución de redes a diferentes niveles.

7. Se estructura en una lógica de intervención territorial y motiva al gobierno para ir cediendo espacios de poder al construir nuevas formas de relaciones con la población.

8. Es flexible, constata la necesidad de una continua adaptación a las diferentes realidades donde se darán las intervenciones.

En la evolución del concepto de desarrollo local, es en 1987 cuando de nuevo toma fuerza el tema a raíz del Informe "Nuestro futuro común", y después con la Cumbre de la Tierra en 1992, en Río de Janeiro, donde con gran respaldo se aprobará la Agenda 21 ("Agenda 21 del Municipio de Sao Paulo". www.google.com.br).

A partir de este momento, el desarrollo local caracterizado por su dimensión territorial, adquiere otra connotación, no solo debido al efecto espacial de los procesos organizativos y 
tecnológicos, sino por el hecho de que cada localidad, cada territorio, es el resultado de una historia en la que se ha ido configurando gradualmente el entorno institucional, económico y organizativo. (“Agenda local 21Motril”. www.agenda local21.es).

El nuevo enfoque del desarrollo local, a partir de la Conferencia de las Naciones Unidas sobre Medio Ambiente y Desarrollo, en junio de 1992, no habla de un desarrollo económico local, sino de desarrollo local sostenible, como aquel:

"promovido y desarrollado por autoridades locales en pro del desarrollo sostenible de su comunidad, para actuar hacia la mejora ambiental del municipio", y como un "proceso donde la forma local de gobierno, ampliamente comunitaria y participativa, tiene por objetivo establecer una exhaustiva estrategia de acción para la protección del medio ambiente, la prosperidad económica y el bienestar social dentro del ámbito local”(pág.6).El Programa de sostenibilidad ambiental “en www.cma.juntaandalucia.es)

En el trabajo de investigación de desarrollo local sostenible liderado por Milagros Morales Pérez (Profesora auxiliar del Departamento de Ciencias Económicas de la Facultad de Ciencias Económicas y Empresariales de la Universidad de Oriente, 2010), fueron objeto de revisión iniciativas internacionales y nacionales, específicamente se consultaron: la experiencia de Seattle, ciudad al Oeste de Estados Unidos; Proyecto de Sostenibilidad que incluye 10 municipios de Gran Bretaña; el municipio Hamilton de Canadá; 15 municipios de España; la experiencia latinoamericana con los casos de Morelia (México), y Machu Picchu (Perú), Córdoba (Argentina), Sao Paulo (Brasil), Costa Rica y como es lógico, la realidad cubana.

En el análisis de las distintas experiencias, se centra la atención en dos momentos claves: los objetivos que persiguen esas localidades para aproximarse a un desarrollo local sostenible y el procedimiento utilizado para lograr sus propósitos. Los objetivos propuestos en las diversas localidades se pueden resumir con las ideas siguientes: cambiar el enfoque en la gestión de la sostenibilidad local del desarrollo, y promover la participación ciudadana en la definición y ejecución de acciones para situar al territorio en la línea de esa nueva concepción del desarrollo.

Si bien las experiencias apuntan, al definir sus intenciones, hacia un desarrollo local sostenible, no todas precisan cuál es el concepto de desarrollo que fundamenta su programa de acción. En esta dirección es de destacar el caso de Córdoba, en Argentina. En esta iniciativa el primer paso consistió en esclarecer qué era la Agenda 21 y después identificar los actores y temas prioritarios para la región.

Las características y propósitos que se hallaron en estas experiencias fueron diversas como: obtener el máximo beneficio en términos no solo económicos, sino también ambientales y sociales, integrar las políticas sociales, económicas y ambientales, bajo criterios de 
sostenibilidad, definir el concepto de sostenibilidad más apropiado para su realidad, dígase "salud y vitalidad cultural, económica y ambiental a largo plazo, la identificación de la concepción de desarrollo solamente con esta arista del proceso socio ambiental.

En la región de Sao Paulo se exponen las etapas que engloba este proceso, que son: sensibilización y compromiso de los órganos y secretarías municipales, capacitación de los funcionarios para la gestión participativa, y compromiso y participación de los sectores sociales “Agenda 21 del Municipio de Sao Paulo", en (www.google.com.br).

En cuanto a la valoración crítica de estas experiencias pueden encontrarse resultados sustanciales en los objetivos propuestos, a partir de la intención de cambiar el enfoque en la gestión de la sostenibilidad local del desarrollo, y promover la participación ciudadana en la definición y ejecución de acciones para situar al territorio en la línea de esa nueva concepción del desarrollo.

No obstante, en opinión del autor de este estudio, se deja un tanto abandonado el tema de la comunicación como proceso que transverzaliza toda la gestión en sus matices y dimensiones, como objetivo central de articulación entre los actores implicados.

El desarrollo local es uno de los elementos más significativos en la creación de redes que favorecen la calidad de vida de los habitantes y el cuidado y protección de los recursos, siendo su eje de transformación, el espacio local, es decir, el espacio en que cada persona puede actuar.

El desarrollo local cuenta con los elementos necesarios para llevar a cabo esa mejora del espacio y la sociedad de la que se le ha hecho protagonista, con la necesidad de que se implique tanto a actores globales como a los agentes y a la población local.

Esto es, en parte, lo que justificaría la consecución de sus fines. Veamos al desarrollo local en cuanto a lo que debe ser y lo que necesita, según la figura 2. 
Figura 2. Elementos del desarrollo local.

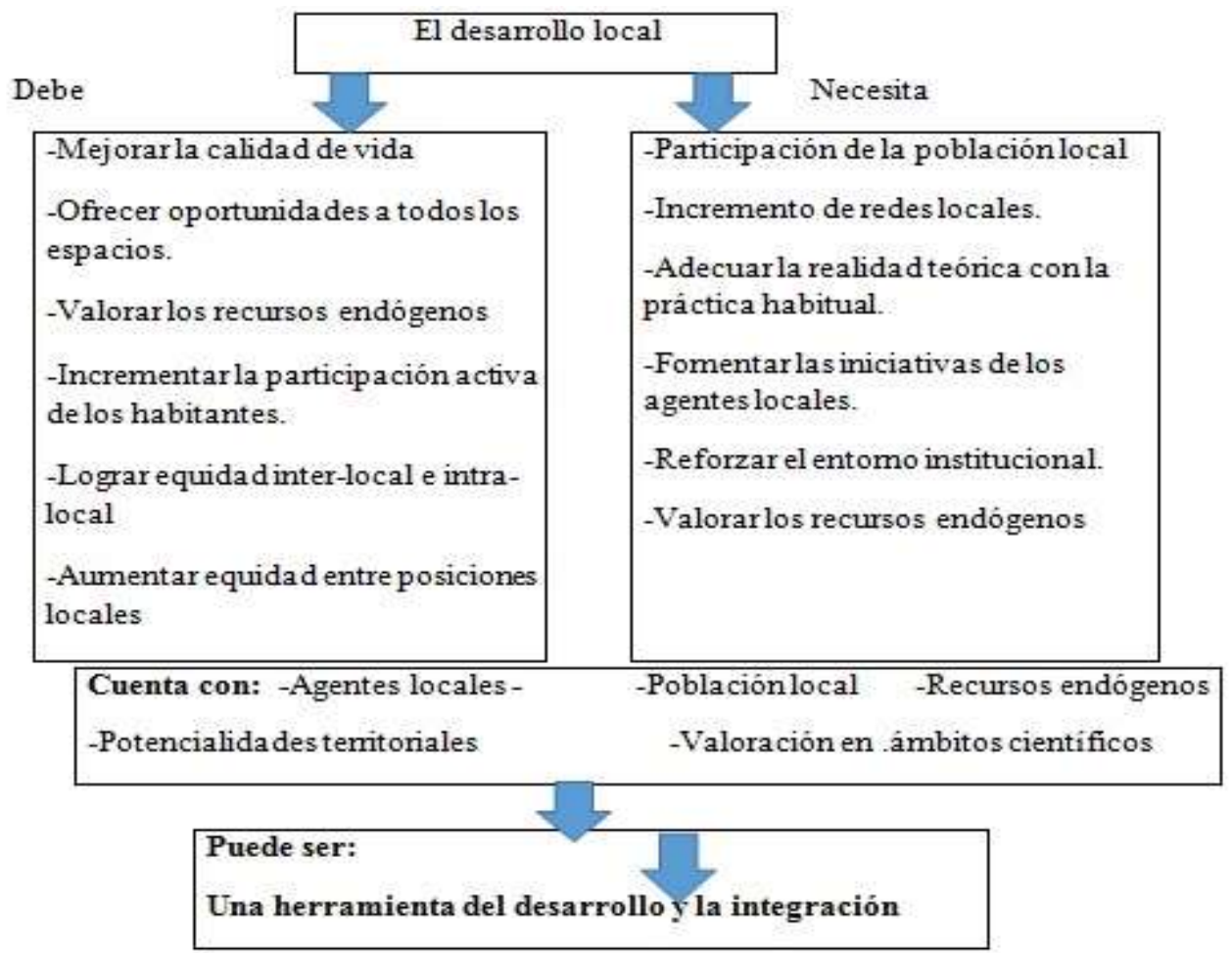

Fuente: Juárez (2013)

El desarrollo local tiene diversos aspectos para ser analizados como el perfil productivo, el tipo de municipio/localidad, los circuitos económicos predominantes, el modelo de gestión, las políticas sociales, y la manera en que se da el mismo proceso.

Todos estos elementos van a marcar hacia dónde va un territorio y que condiciones tiene para generar crecimiento económico con inclusión social, es decir, desarrollo local. La interrelación o sinergia de las dimensiones del desarrollo territorial resulta esencial.

Una de las condiciones claves para el desarrollo local, para su materialización, es la constatación de los objetivos de desarrollo local. El objetivo general que se propone en el ámbito de desarrollo local es la transformación del sistema económico y social de una localidad. Así lo muestra la figura 3: 
Figura 3. Interrelación o sinergia de las dimensiones del desarrollo territorial.

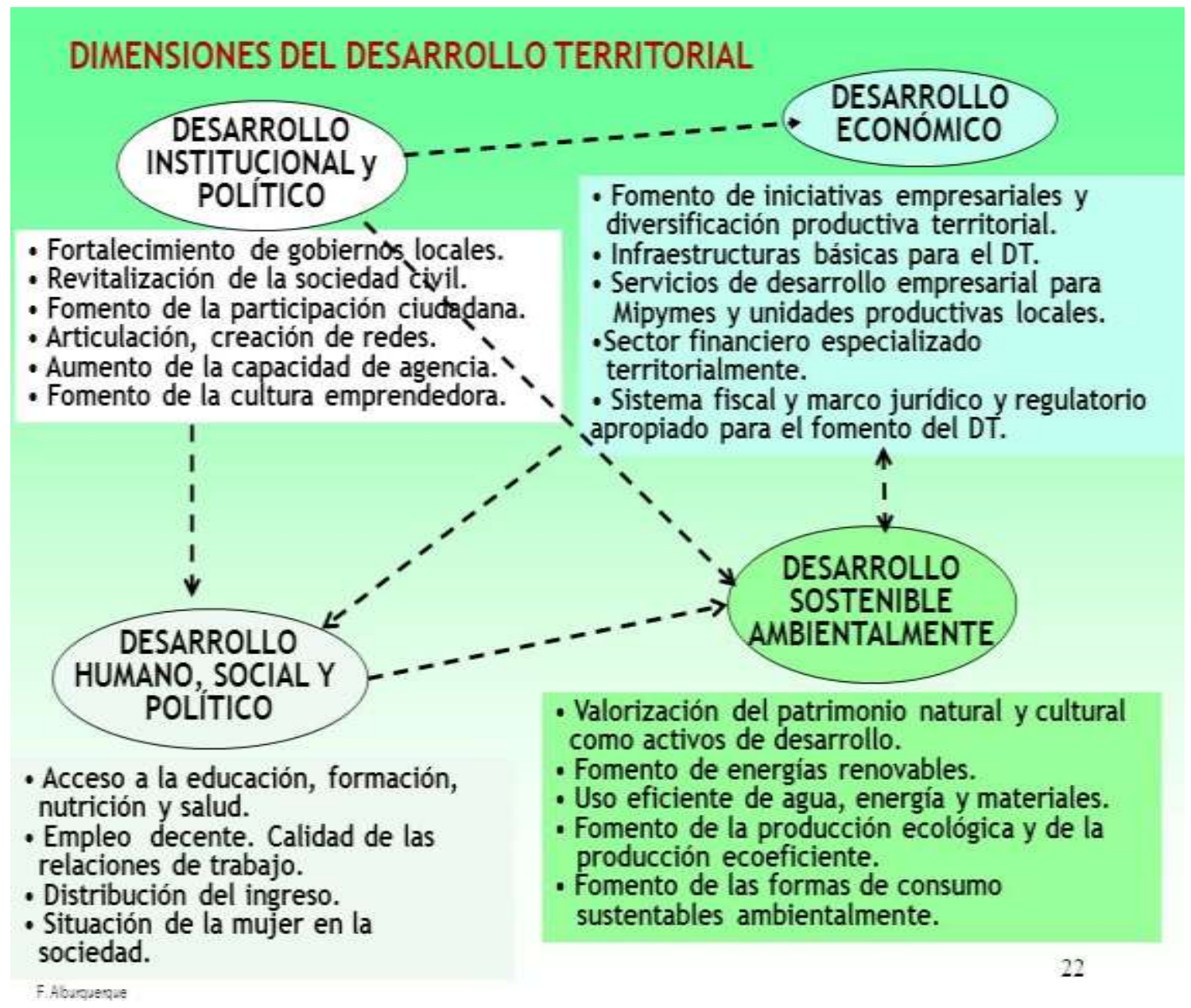

Fuente: Alburquerque (2008)

Para el autor de este estudio, a partir de criterios de otros autores, como Coraggio (1991), Rodríguez (1999),Hernández (2006),Colectivo de Autores (2011-2015) y desde la mirada de proceso de toda la vida, se debe entender la transformación social principalmente como un proceso que nunca acaba, que las metas se van corriendo con la historia y que habría que definir cómo se piensa ese proceso.

En todo caso, en su base va a incluir la estructuración o reestructuración continua, la configuración o reconfiguración del conjunto de las distintas clases, de los estamentos de las identidades colectivas, las identidades de las capas profesionales, los sistemas de integración, las comunidades de distintos tipos de etnias vecinales, regionales, ideológicas; la relación entre Estado y ciudadanía, los sistemas de regulación de los conflictos, el imaginario social no solo en determinados contextos donde interactúan varias comunidades, sino el de las propias comunidades insertadas en un mismo contexto dentro de un Consejo Popular. 
Tal es el caso del estudio que se lleva a cabo, la cual se desarrolla en los Consejos Atares El Pilar en el Municipio El Cerro, con su propio orden de prioridades dentro de sus necesidades sentidas reconocidas o no. Todo esto ratifica y tributa a los resultados que esa estructura en cambio permite, lo que tiene mucho que ver con la llamada transformación social.

El desarrollo local en el espacio de una comunidad permite crear dinámicas nuevas, relacionar sectores diversos, coordinar instituciones, articular actores varios. Es, por lo tanto, este abordaje inicial el que permite definir las unidades de intervención pertinentes, ubicar las localidades e identificar las comunidades.

Para Velázquez (1998), citado en Carbajal (2011), el desarrollo local es aludir al conjunto de procesos económicos, sociales, culturales, políticos y territoriales mediante los cuales una comunidad, a partir de sus propias potencialidades y de las oportunidades que brinda el entorno, accede al bienestar sin exclusiones, ni discriminaciones y garantiza las condiciones para que las futuras generaciones puedan hacerlo.

Se utilizaron los siguientes:

\section{Métodos de investigación, Técnicas e Instrumentos:}

\section{Teóricos}

- Histórico-lógico: Lo histórico se refiere al estudio del objeto en su trayectoria real a través de su historia. Lo lógico interpreta lo histórico e infiere conclusiones. De la Uz, Lemus, Valdés y Padrón (2010). Se aprecia en la descripción del desarrollo local, la su origen, conceptualizaciones, principales características y dimensiones.

- Analítico-Sintético: El análisis es un procedimiento lógico que posibilita descomponer mentalmente un todo en sus partes y la síntesis es la operación inversa, que establece mentalmente la unión o combinación de las partes previamente analizadas Martínez (2016). Se aprecia en el empleo de la elaboración de los fundamentos teóricos y metodológicos en que se sustenta el estudio, así como en el procesamiento de la información y para la interpretación de los resultados.

- Inductivo- deductivo: La inducción es una forma de razonamiento en la que se pasa del conocimiento de casos particulares a un conocimiento más general y la deducción se pasa de un conocimiento general a otro de menor nivel de generalidad Dávila (2006). Se aprecia en la determinación de los postulados generales que permiten la formulación del problema, y la caracterización del desarrollo local.

\section{Empíricos}

- Análisis documental. La revisión de los documentos normativos de desarrollo local en Cuba que pueden tributar al estudio y trabajo de trasformación social para el diagnóstico. 
En este estudio el autor pudo investigar y posicionar un seguimiento a los autores que trabajan el tema, así como que, constató lo importante del vínculo de la gestión del conocimiento en las estructuras de gestión del desarrollo local y su mirada interdisciplinaria como sustento teórico, el cual le permitió ordenar y tributar elementos importantes y conceptuales al desarrollo desde una realidad constatada en el ámbito local, con particularidades y dinámicas de gestión muy propias que identifican la cultura local. Esto le permitió identificar al desarrollo como un proceso que se apoya en las fuerzas endógenas como iniciativas que impulsan una identidad propia dentro del sistema, y que busca el reconocimiento de la importancia del territorio a nivel local. Unido a esto el elemento de la formación educativa y la comunicación pasan a ser factores esenciales para desarrollar la capacidad de innovación y la creatividad, a la vez que la integración y la solidaridad en el desarrollo local.

\section{Conclusiones.}

- Para el autor de este estudio, es vital acotar como primera conclusión, su mirada conceptual con respecto al desarrollo local, el cual lo define como un proceso de crecimiento y cambio estructural de la economía de una ciudad, comarca o región, en el que se pueden identificar, al menos, tres dimensiones: económica, caracterizada por un sistema de producción que permite a las empresas locales usar eficientemente los factores productivos, generar economías de escalas y aumentar la productividad a niveles que permitan mejorar la competitividad en los mercados; sociocultural, en que el sistema de relaciones económicas y sociales, las instituciones locales y los valores, sirven de base al proceso de desarrollo, y político-administrativa, en que las iniciativas locales crean un entorno local favorable a la producción e impulsan el desarrollo sostenible a partir de sus propias realidades que los actores implicados definen.

- De todos los conceptos de desarrollo local, abordados, el de Velázquez (1998), citado en Carbajal (2011), es con el que, el autor de ese estudio se identifica de manera vivencial, ya que, si se revisa este concepto a profundidad, inmediatamente se puede discernir hacia donde se debe trabajar, y cómo el trabajo de gestión sociocultural dentro del desarrollo local no puede seguir siendo un conjunto de acciones para cumplir un plan, pensando que aquí está el éxito.

- No se debe seguir pensando en lo que se hizo bien o mal, se trata de lo que se debe hacer y cómo hacerlo bien, sin equivocaciones, y tratando siempre de que todos los procesos sean integradores y articuladores, y estén dirigidos a la inclusión y la participación de todos los actores, con una inyección de sentido de pertenencia, siendo sujetos protagónicos, con una identidad local, que sería la única forma de comprometer a las comunidades con su propio desarrollo y transformación social desde su realidad constatada. 


\section{Referencia bibliográfica}

"Agenda 21 del Municipio de Sao Paulo", www.google.com.br

"Agenda local 21 Motril". www.agendalocal21.es

Arocena, J. (2002). El desarrollo local: un desafío contemporáneo. Uruguay: Taurus. Universidad Católica. Segunda Ed.

Colectivo de autores. (2011-2015). Prodel: Programa Cuatrienal para el fortalecimiento de las capacidades municipales. CEDEL-INIE-CIERIC-CUBAENERGIA-INIFATGUCID, La Habana.

Colectivo de autores, D. (2011). Cataurito de experiencias para el desarrollo local. La Habana: Caminos.

Cotorruelo M; Vázquez Barquero, A (1995): "Notas sobre la estrategia de Desarrollo Territorial"; Cuadernos de Aguilar N ${ }^{\circ}$ 5. Instituto Nacional de Empleo - Fundación de la Universidad Autónoma de Madrid. Madrid (España)

Coraggio, J. L. (1991). Ciudades sin Rumbo. Investigación urbana y proyecto popular. SIAP / Ciudad, Quito.

Corvalan, E y Ferreira M (2003). Desarrollo local. Una metodología para la participación. Santiago de Chile, LOM Ediciones.

Dollfus, O. (1997). La mundialización. París: Presses da Sciences Post

Hernández, JL (2006): La ciencia y la Tecnología como factores de desarrollo local. El espacio local. En: Guzón, a. (comp.)Desarrollo local en Cuba. Retos y perspectivas. La Habana, Editorial Academia. 175-184.

Llobera Serra, F. (2001). "Conceptos clave, dinamización y asistencia en programas de desarrollo rural". En: Lecciones de Desarrollo Rural. Ciudad Real: Universidad de Castilla-La Mancha, CEDERCAM. 51-70.

Rodríguez, F. (1999): Manual de Desarrollo Local. Gijón, Ed. Trea,

Silva (2006) citada en Carbajal(2011):enJuárez Alonso GRecibido: Marzo, 2012 // Aceptado:

Abril, 2013. Revisión del concepto de desarrollo local desde una perspectiva territorial.Revista Líder Vol. 23. 2013 - pp 9-28 ISSN: 0717-0165 versión impresa, ISSN: 0719-5265 versión en línea. PDF.

Touraine, A. (1995). Producción de la sociedad. México: UNAM. 
Unión Europea, U. E. (2014). “Fondos Estructurales y de Inversión Europeos. Orientaciones para los Estados miembros y las Autoridades del Programa. Orientaciones para los Beneficiarios" Orientaciones sobre desarrollo local a cargo de las comunidades locales para los agentes locales.

UNESCO (2008): Declaración de la Conferencia Regional de Educación Superior para América Latina y el Caribe, Cartagena de Indias.

Valdizán García, Mª I. (2007). Universidad de Burgos y desarrollo local. Aproximación a un modelo teórico centrado en el desarrollo local desde la Universidad. (Tesis doctoral no publicada). Universidad de Burgos. Facultad de Humanidades y Ciencias de la Educación.

Velázquez (1998), citado en Carbajal (2011), en Juárez Alonso G Recibido: Marzo, 2012 // Aceptado: Abril, 2013. Revisión del concepto de desarrollo local desde una perspectiva territorial. Revista Líder Vol. 23. 2013 - pp 9-28 ISSN: 0717-0165 versión impresa, ISSN: 0719-5265 versión en línea. PDF.

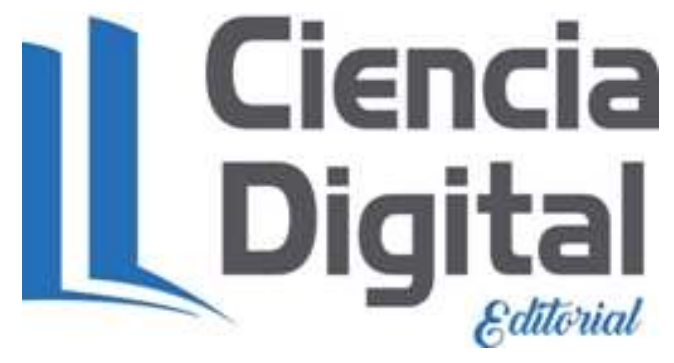




\section{Para citar el artículo indexado.}

Santa Cruz D, Ojalvo V. \& Velasteguí E. (2019) Desarrollo local: conceptualizaciones, principales características y dimensiones. Revista electrónica Ciencia Digital 3(2), 319335. Recuperado desde:

http://cienciadigital.org/revistacienciadigital2/index.php/CienciaDigital/article/view/353/907

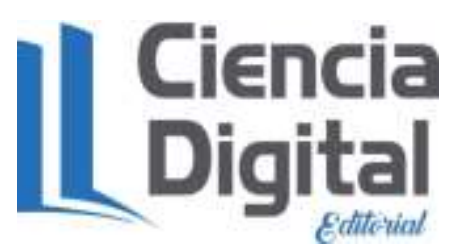

El artículo que se publica es de exclusiva responsabilidad de los autores y no necesariamente reflejan el pensamiento de la Revista Ciencia Digital.

El articulo queda en propiedad de la revista y, por tanto, su publicación parcial y/o total en otro medio tiene que ser autorizado por el director de la Revista Ciencia Digital.
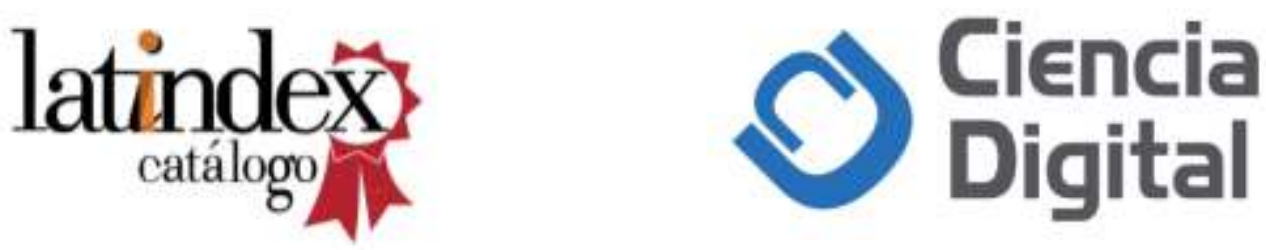